\title{
Human CIK Cells Loaded with Gold Nanoprisms as Theranostic Platform for Targeted Photoacoustic Imaging and Enhanced Immuno-Photothermal Combined Therapy
}

\author{
Jingjing Zhang ${ }^{1}$, Fangfang Xia ${ }^{1}$, Yao Yang ${ }^{1}$, Caixia Yue ${ }^{1}$, Chunlei Zhang ${ }^{1}$, Yuming Yang ${ }^{1}$ \\ Lijun $\mathrm{Ma}^{3}$, Gabriel Alfranca ${ }^{1}$, Yanlei Liu ${ }^{1}$, Yafei Hou ${ }^{1}$, Weilin Jin ${ }^{1}$, Jian $\mathrm{Ni}^{1}$ \\ Jesus Martinez de la Fuente ${ }^{1,4}$, Daxiang Cui ${ }^{1,2,3}$
}

\begin{abstract}
${ }^{1}$ Institute of Nano Biomedicine and Engineering, Shanghai Engineering Center for Intelligent Diagnosis and Treatment Instrument, Department of Instrument Science and Engineering, School of Electronic Information and Electrical Engineering, Shanghai Jiao Tong University, 800 Dongchuan Road, Shanghai 200240, P. R. China.

${ }^{2}$ National Center for Translational Medicine, Collaborative Innovational Center for System Biology, Shanghai Jiao Tong University, 800 Dongchuan Road, Shanghai 200240, P. R. China.

${ }^{3}$ Department of tumor, Shanghai Tong Ren Hospital attached to Shanghai Jiao Tong university, 786 Yuyuan Road, Shanghai 200050 , P. R. China.

${ }^{4}$ Instituto de Nanociencia de Aragon (INA), Universidad de Zaragoza, Zaragoza, 50018, Spain,
\end{abstract}

Corresponding authors. E-mail: yumingyang@sjtu.edu.cn, dxcui@sjtu.edu.cn

Received: Jun. 29, 2016; Accepted: Jul. 4, 2016; Published: Jul. 11, 2016.

Citation: Jingjing Zhang, Fangfang Xia, Yao Yang, Caixia Yue, Chunlei Zhang, Yuming Yang, Lijun Ma, Gabriel Alfranca, Yanlei Liu, Yafei Hou, Weilin Jin, Jian Ni, Jesus Martinez de la Fuente and Daxiang Cui, Human CIK Cells Loaded with Gold Nanoprisms as Theranostic Platform for Targeted Photoacoustic Imaging and Enhanced Immuno-Photothermal Combined Therapy. Nano Biomed. Eng., 20 I6, 8(3): I I2-I 27. DOI: 10.5101/nbe.v8i3.p112-127.

\begin{abstract}
How to enhance the therapeutic efficacy of human cytokine induced killers cell (CIK) has become a great challenge. Herein, we report for the first time that human CIK cells loaded with gold nanoprisms were successfully used for targeted photoacoustic imaging, enhanced immunotherapy and photothermal therapy of gastric cancer in vivo. Gold nanoprisms were synthesized and modified with PEG; human CIK cells were prepared and incubated with PEGylated gold nanoprisms (Au GNPrs), and then the effects of human CIK cells labeled with Au NPrs on gastric cancer MGC 803 cells were evaluated and further used for targeted photoacoustic imaging, immunotherapy and photothermal therapy of gastric cancer in vivo in mice models. Results showed that PEGylated Au NPrs could be uptaken high-efficiently by human CIK cells, resultant human CIK cells labeled with AuNPrs could inhibit the growth of gastric cancer MGC 803 cells actively by induced apoptosis and G1 phase arrest, and actively target and accumulate the tumor sites in gastric cancer-bearing nude mice. Enhanced synergistic therapeutic efficacy was demonstrated with the maximal inhibition of tumor through a combination of CIK cells-based immunotherapy for three days and then a continuous gold nanoprisms-based photothermal therapy. In conclusion, human CIK cells labeled with PEGylated $\mathrm{Au}$ NPrs can target gastric cancer cells in vivo, enhance immunotherapy and photothermal therapy efficacy, and have a great potential in applications such as targeted imaging, immunotherapy and photothermal therapy of gastric cancer in the near future.
\end{abstract}

Keywords: Gold nanoprism; CIK cell; Gastric cancer; Targeted imaging; Photoacoustic imaging; Photothermal therapy; Cytokine factors 


\section{Introduction}

Stomach cancer (GC) is the fourth most common cancer and the second leading cause of cancer-related death worldwide [1]-[3]. It ranks No. 2 among all malignant tumors in China according to the latest cancer disease spectrum [4]. It has been proven that the traditional therapeutic strategies including surgery, chemotherapy and radiotherapy are not effective enough to eradicate GC cell completely, and that metastasis and recurrence are the major causes of death. The gastric cancer prognosis is very poor with the 5-year survival rate below $24 \%$ [5]. How to improve the therapeutic efficacy of GC has become a key scientific problem.

In the recent years, tumor immunotherapy has become a hotspot. Especially dendritic cells (DCs) and cytokine induced killer (CIK) cells have been broadly used for clinical therapy of post-operation tumor patients, and achieved better therapeutic efficacy [6]-[9]. CIK cells are a heterogeneous subset of exvivo expanded T lymphocytes [10]-[12]. They present a mixed T-NK phenotype and are endowed with the MHC-unrestricted antitumor activity, which means it can kill cancer cells without attacking the normal cells $[13,14]$. Although CIK cells have the ability to kill cancer cells, the curative effect is not obvious in a short time. Current reports show that CIK cells own the ability to target in vivo tumor cells $[15,16]$, homing to the tumor site through vascular perfusion. And the targeting characteristic of CIK cells has an extensive adaptability feature, which can be applied to clinical therapies of many kinds of cancers. However, up to date, few reports have demonstrated that tumor patients with middle and late stages can be cured completely by using DCs and CIK immunotherapies [17]-[22]. How to enhance CIK therapeutic effects has become a challengeable problem. In addition, up to date it is not well clarified what is the mechanism of the bio-distribution and tracking of CIK cells in vivo. Therefore, it is very necessary to develop new therapeutic strategies that could enhance CIK therapeutic effects and/or improve synergistic combined therapy.

Up to date, nanoparticles-based theranostics has achieved great advances in cancer prevention, imaging and therapy [23]-[26]. It not only provides excellent capacity of carrying multiple theranostic agents in the same package, but also facilitates targeting delivery into specific sites and across complex biological barriers [27, 28]. For example, gold nanoparticles (GNPs) have drawn much more attention due to their unique optical properties and the potential applications. Up to date, various shapes of GNPs have been prepared, such as gold nanospheres, gold nanorods, gold nanoprisms (Au NPrs), gold nanostars, gold nanoshells, and gold nanowires [29]-[31], which have determined their different applications such as photothermal therapy (PTT) [32]-[34], photoacoustic imaging [35]-[37] and surface enhanced resonance spectroscopy (SERS) [38]. Especially, gold nanoprisms (AuNPrs) have a symmetrical triangle structure, and a relative bigger specific surface area to display an obvious surface plasmon resonance (SPR) band in the near-infrared (NIR) region of the electromagnetic (EM) spectrum and can be excited to produce heat efficiently $[39,40]$. More importantly, the NIR radiation has a deeper penetration depth in biological tissues, which makes it an excellent theranostic agent for medical applications such as photoacoustic imaging and photothermal therapy (PTT). In our previous work, we prepared PEG modified gold nanoprisms and realized photoacoustic imaging of gastrointestinal tumors [41]. We also prepared gold nanorods and gold nanostars, and realized targeted photoacoustic imaging and PTT of gastric cancer in nude mice models $[42,43]$. Due to that gold nanoprisms own good biocompatibility and stability in vivo, we consider that gold nanoprisms can be controllably synthesized and easier for clinical translation $[44,45]$.

In this study, we took full advantages of human CIK cells and gold nanoprisms, with the aim of enhancing synergistic CIK cell therapeutic effects combined with PTT of gold nanoprisms. We prepared human CIK cells, synthesized CD3 antibody-conjugated PEG modified gold nanoprisms, and then prepared human CIK cells labeled with $\mathrm{Cd} 3$ antibody conjugated PEGylated gold nanoprisms, and investigated the in vivo distribution and tumor targeting ability and the PTT therapeutic efficacy. The results show that human CIK cells labeled with CD3 antibody-conjugated PEGylated gold nanoprisms could target gastric cancer in vivo, realize photoacoustic imaging and photothermal therapy of gastric cancer, and markedly enhance CIK immunotherapeutic efficacy. Our results provide a new strategy to treat gastric cancer, and offer proof-of-principle of practicality of CIK combined with PTT based on human CIK cells labeled with CD3 antibody conjugated PEGylated gold nanoprisms, 
exhibiting potential clinical applications such as theranostics of gastric cancers in middle and late stages and post-operation tumor patients in near future.

\section{Experimental}

\section{Materials and Methods}

\section{Materials}

Gold (III) chloride trihydrate $\left(\mathrm{HAuCl} 4 \cdot 3 \mathrm{H}_{2} \mathrm{O}\right.$, 99\%) and sodium thiosulfate were obtained from Sinopharm Chemical Reagent Co., Ltd, China. For the derivatization of AuNPrs with polyethylene glycols (PEG), HS- $\mathrm{C}_{2} \mathrm{H}_{4}$-CONH-PEG-O- $\mathrm{C}_{3} \mathrm{H}_{6}$-COOH (HSPEG-COOH, M.W. $5000 \mathrm{~g} \mathrm{~mol}^{-1}$ ) was purchased from Rapp-Polymere. All glassware was washed with aqua regia and rinsed thoroughly with ultrapure water (Milli-Q grade). Annexin V-FITC/PI Apoptosis Detection Kit and Cell Counting Kit (CCK-8) were obtained from Yeasen Corporation (Shanghai, China). MGC-803 cell lines were available in the Cell Bank of Type Culture Collection of Chinese Academy of Sciences. Cell culture products were purchased from GIBCO.

\section{Preparation and characterization of PEGylated AuNPrs}

The PEG modified gold nanoprims were synthesized according to our previous report [46]. First, we mixed $10 \mathrm{ml}$ of $2 \mathrm{mM}$ chloroauric acid and $12 \mathrm{~mL}$ of $0.5 \mathrm{mM}$ sodium thiosulfate (reducing regent) attired gently for 9 mins at room temperature. Then $2 \mathrm{~mL}$ of the sodium thiosulfate was added to the above solution again after the colloidal solution changed from yellow to brown. 90 mins later, the reaction was completed. To enhance the colloidal purity and stability of the products, the AuNPrs were modified with polyethylene glycols (PEG), HS- $\mathrm{C}_{2} \mathrm{H}_{4}$-CONH-PEG-O- $\mathrm{C}_{3} \mathrm{H}_{6}$ - $\mathrm{COOH}$ (HSPEG-COOH, M.W. $5000 \mathrm{~g} \mathrm{~mol}^{-1}$ ). $10 \mathrm{ml}$ of GNPs was supplemented with $1 \mathrm{mg}$ of PEG. The $\mathrm{pH}$ value of the solution was adjusted to 12 with $\mathrm{NaOH}$, and it was stired overnight. The solution was centrifuged at $6000 \mathrm{rpm}$ for 3 times, and PEG modified gold nanoprisms were obtained. Prepared gold nanoprisms and PEG modified Au NPrs were characterized by high resolution TEM and UV-vis absorption spectra.

\section{Preparation and identification of human CIK cells}

Human CIK cells were expanded from peripheral blood collected from the healthy people in our
Laboratory [17]-[22]. All individuals provided informed consent for blood donation according to a protocol approved by the internal review board and ethic committee. First, we separated the peripheral blood mononuclear cells (PBMC) by density gradient centrifugation (Lymphoprep, Axis-Shield); second, we seeded them in cell culture flasks at a concentration of $1 \times 10^{6}$ cells $/ \mathrm{mL}$ in RPMI-1640 medium (Gibco BRL) only and added the IFN-g (PeproTech; 1000 $\mathrm{U} / \mathrm{mL}$ ). After $24 \mathrm{hrs}$, interleukin (IL)-2 (PeproTech,) and anti-CD3 antibody (OKT3, Abgent) were added at a concentration of $300 \mathrm{U} / \mathrm{mL}$ and $50 \mathrm{ng} / \mathrm{mL}$, respectively. Fresh medium and IL-2 $(300 \mathrm{U} / \mathrm{mL})$ were added one time every 3 days during culturing, and the cell concentration was maintained at $1 \times 10^{6}$ cells/ $\mathrm{mL}$. On the 14th day, the composition and purity of CIK cells were assessed by FACS (FACS Calibur, BD Biosciences, USA) with FITC-CD3, and PE-CD56, and then the cells and the culture supernatant were harvested for later use.

\section{Preparation and characterization of CIK cells labeled with PEGylated AuNPrs}

We prepared $1 \times 10^{6} \mathrm{CIK}$ cells incubated with 1 $\mathrm{ml}$ of $100 \mu \mathrm{g} \mathrm{mL}{ }^{-1}$ PEGylated AuNPrs in $37{ }^{\circ} \mathrm{C}(5 \%$ $\mathrm{CO}_{2}$ ) carbon dioxide incubator for $24 \mathrm{hrs}$. Then we collected CIK cells after washing them three times in PBS at $1000 \mathrm{rpm}$. Cells were fixed on coverslips with 4\% paraformaldehyde solution for 20 mins at room temperature by StatSpin CytoFuge Cytocentrifuge. Two-photon laser scanning microscopy was used to observe CIK cells by using a confocal microscope (Olympus FV 1000) with excitation at $810 \mathrm{~nm}$. CIK cells incubated with Cd56-AuNPrs for 6, 12 and 24 hrs at $37^{\circ} \mathrm{C}$, and were then collected. For each sample, the number of the cell count up to $2 \times 10^{7}$. Then the $\mathrm{Au}$ content was analyzed using ICP-MS (NexION300X, Perkinelmer).

\section{Effects of PEGylated AuNPrs on CIK cell viability}

Cell viability was determined by Cell Counting Kit (CCK-8) assay. MGC-803 cells / CIK cells were seeded at a density of $6 \times 10^{3}$ in a 96-well plate (Corning, Costar, NY) for $24 \mathrm{hrs}$ before and incubated with various concentrations of PEGylated AuNPrs for the next 12 hrs. After exposure, cells were washed by PBS for one time. Then we added $100 \mu$ DMEM for MGC803 cells (RPMI-1640 medium for CIK cells) and $10 \mu \mathrm{lCCK}-8$ to each well. After $2 \mathrm{hrs}$, the 96well plate was measured at $570 \mathrm{~nm}$ using a standard 
micro plate reader (Scientific Multiskan MK3, thermo, USA). The cell viability was calculated according to the formula: cell viability $=(\mathrm{OD}$ of the experimental sample/OD of the control group) $\times 100 \%$.

\section{Effects of CIK cells labeled with PEGylated Au NPrs on MGC 803 cells}

Effects of PEGylated Au NPrs-labeled CIK cells on MGC 803 cells were investigated by cell cycle analysis and apoptosis assay. The MGC-803 cells were co-incubated with gold nanoprims-labeled CIK cells based on various ratio: 20:1, 40:1 and various incubation time: $12 \mathrm{hrs}, 24 \mathrm{hrs}$ in 24-well plate. Then all the cells were collected, and resuspended in $150 \mu \mathrm{l}$ binding buffer. Then $5 \mu \mathrm{l}$ of Annexin V-FITC and 1.5 $\mu l$ of PI were added and incubated in the dark at room temperature for 15 mins. Lastly, $400 \mu 1$ PBS was added to each sample, the apoptotic and necrotic distribution of MGC 803 cells was analyzed immediately by flow cytometry.

Cell cycle analysis was carried out by PI staining followed by flow cytometric measurement. Cells were prepared as above mentioned. After the cells were collected, $4{ }^{\circ} \mathrm{C} 70 \%$ ethanol were added to fix them overnight at $-20{ }^{\circ} \mathrm{C}$. Then remove the ethanol and wash the cells with PBS for once. Then the cells were stained with $50 \mu \mathrm{g} / \mathrm{ml}$ PI and incubated in the dark at room temperature for $0.5 \mathrm{hr}$, followed by an analysis with a FACSCalibur (BD Biosciences, Mountain View, CA).

\section{CIK cells labeled with PEGylated AuNPrs cells for targeted photoacoustic imaging and immunotherapy}

All animal experiments were approved by the Institutional Animal Care and Use Committee of Shanghai Jiao Tong University (SCXK-2012-0002). First, subcutaneous gastric cancer-bearing nude mice models were prepared. The human gastric cancer cell MGC-803 were cultured at $37{ }^{\circ} \mathrm{C}\left(5 \% \mathrm{CO}_{2}\right)$ carbon dioxide incubator in Dulbecco's Modified Eagle's Medium (DMEM, HyClone) consisting of $10 \%$ (vol/ vol) fetal bovine serum (FBS, Gibco), $100 \mathrm{U} / \mathrm{mL}$ penicillin and $1 \mathrm{mg} / \mathrm{mL}$ streptomycin. A total of 20 C57BL/6 mice were used to establish subcutaneous GC models with MGC-803 cells by injecting MGC803 cells $\left(1 \times 10^{6}\right)$ into the right anterior flank area of male nude mice aged 4 to 5 weeks. Four weeks later, tumors grew to approximately $5 \mathrm{~mm}$ in diameter, and then were used for experiments.
PEGylated AuNPrs-labeled CIK cells were used for fluorescence imaging in vivo. We tracked the distrbution of CIK cells in vivo by using fluorescent marker DiR (one kind of near infrared dyes, Ex/ Em748/780) embedded in the cytomembrane of cells. $1 \times 10^{6} \mathrm{CIK}$ cells were harvested and incubated with $4 \mu 1$ of $2.5 \mathrm{mmol} \mathrm{DiR}$. After 20 mins incubation, the sample was washed for two times, then injected into the mice via the tail vein. Mice were monitored and photographed by IVIS Lumina imaging system (Xenogen) at 1, 3 and 7 days respectively.

PEGylated AuNPrs labeled CIK cells were used for photoacoustic imaging of gastric cancer-bearing nude mice model. Gastric cancer-bearing nude mice were divided into three groups, control no.1 group (only injection of PBS), control no. 2 group (only injection of AuNPrs $(200 \mu \mathrm{g} / \mathrm{mL})$ ), experiment group (Au NPrlabeled CIK, $1 \times 10^{6} \mathrm{CIK}$ cells incubated with $200 \mu \mathrm{g} /$ $\mathrm{mL}$ for $24 \mathrm{hrs}$ ), which were respectively injected with PBS, AuNPrs and PEGylated AuNPrs-labeled CIK cells via tail veins into gastric cancer-bearing nude mice. The images were collected at pre-injection, and 6, 12, 24 and $72 \mathrm{hrs}$ post-injection time points for excitation at $800 \mathrm{~nm}$ by NEXUS 128 photoacoustic imaging system. Then, the mouse sera at $72 \mathrm{hrs}$ postinjection were collected from control group and test group, and sent for commercial service company to detect the concentration of cytokine factors such as GM-CSF, IFN-g, IL-1b, IL-13, IL-2, IL-4, IL-5, IL-6, TNF-a, IL-10, IL-22 and IL-27.

\section{CIK cells labeled with CD56-AuNPrs for photothermal therapy}

The nude mice model with subcutaneous gastric cancer were prepared as described above. The test groups included untreated group, test 1 group $\left(1 \mathrm{~W} / \mathrm{cm}^{2}\right.$ laser irradiation only), test 2 group (CIK only), test 3 group (CIK loaded with PEGylated Au NPrs only) and test 4 group (CIK loaded with PEGylated Au NPrs +1 $\mathrm{W} / \mathrm{cm}^{2}$ laser). For photothermal experiments, the 808 $\mathrm{nm}$ laser trigger was setup as $30 \mathrm{~W} / \mathrm{cm}^{2}$. The tumor sites were positioned in front of the beam, temperature was monitored in real time by a thermometer. after 3 days being injected when most of the CIK cells loaded with PEGylated AuNPrs came to the tumor site, laser irradiation $\left(1 \mathrm{~W} / \mathrm{cm}^{2}\right)$ was done in the tumor site for 2 mins maintaining the temperature to $45^{\circ} \mathrm{C}$. The tumor volumes were calculated over 5 weeks, the tumor growth weekly was monitored with a caliper and the tumor volume was calculated according to the formula: $\mathrm{V}=4 / 3 \times \pi \times(\mathrm{a} / 2)^{2} \times(\mathrm{b} / 2)$, where $\mathrm{a}$ is the length, 
and $b$ is the width diameter of the tumor; the tumor growth curve was drawn. The mouse sera from test 4 group were collected and sent for commercial service company to detect the concentration of cytokine factors such as GM-CSF, IFN-g, IL-1b, IL-13, IL-2, IL-4, IL5, IL-6, TNF-a, IL-10, IL-22 and IL-27.

\section{In vivo distribution and pathological analysis of CIK cells labeled with PEGylated AuNPrs}

MGC803 cells $\left(5 \times 10^{6}\right)$ were injected subcutaneously into the right back flank area of 6 to 8 weeks old female nude mice. Tumors were allowed to grow to a diameter of approximately $5 \mathrm{~mm}$. At that point, $2 \times 10^{6}$ CIK cells labeled with PEGylated AuNPrs were injected into the mice via the tail vein. 12 mice were divided into four group and were then sacrificed 1, 3, 5 , and 7 weeks later. Several hundreds of microliter blood were immediately collected from the heart, mixed with $1 \mathrm{mg}$ EDTA in microfuge tubes and frozen in liquid nitrogen. Then, organs were collected and frozen in liquid nitrogen. Blood and tissue samples were completely lysed in aqua regia, followed with the evaporation process. The resulting precipitates were dissolved in $0.5 \mathrm{M} \mathrm{HCl}$. The Au content determination was conducted on an Agilent 7500a and every data point was expressed as a mean $\pm \mathrm{SD}$ from triplicate samples. Data were represented as \% of injection dose/ organ.

Mice were treated with human CIK cells labeled with PEGylated AuNPrs for 7 days, and then were sacrificed, Heart, liver, spleen, lung, kidney, intestinal tissues and brain were removed and then fixed in $4 \%$ neutral buffered formalin for at least 1 day. The samples were then dehydrated in an ethanol series, processed into paraffin and sectioned. Then, H\&E (hematoxylin and eosin) staining was carried out in accordance with the standard protocol to monitor the morphological features of each organ.

\section{Statistical analysis}

Each experiment was repeated three times in duplicate. The results were presented as mean \pm SD. Statistical differences were calculated by a two-tailed, unpaired Student's t-test at a significance level of $p<$ 0.05 .

\section{Resuls and Discussion}

\section{Preparation and characterization of PEGylated AuNPrs}

The gold nanoprisms (AuNPrs) were synthesized according to previous report [46], a straightforward method without any toxic reagents used, such as cetyltrimethylammonium bromide (CTAB). $10 \mathrm{~mL}$ of $2 \mathrm{mM}$ chloroauric acid (HAuC14) and $12 \mathrm{~mL}$ of freshly prepared $0.5 \mathrm{mM}$ sodium thiosulfate (Na2S2O3) were mixed, and stirred at $30{ }^{\circ} \mathrm{C}$ for 9 mins. The solution color changed gradually from yellow to brownish, the "seeds" were formed. Then there were a second addition of $\mathrm{Na}_{2} \mathrm{~S}_{2} \mathrm{O}_{3}$ in the same conditions. Interestingly, the amount of the second reducing agent contributed to the different location of major peak in UV-Vis-NIR spectrum. There were two distinctive plasmon bands of colloidal solution. One was at 530 $\mathrm{nm}$, which corresponds to the out-plane dipolar mode and is the characteristic absorption peak of $\mathrm{Au}$. The other was at 700-1000 nm, which corresponds to the in-plane dipolar mode; the region of NIR is the major peak of this colloidal solution. As shown in Figure $1 \mathrm{~b}$, the UV-Vis-NIR spectra showed different redshifts as the amount of the second addition of $\mathrm{Na}_{2} \mathrm{~S}_{2} \mathrm{O}_{3}$ increased. In this study, we finally used $2 \mathrm{~mL}$ of the second addition of $\mathrm{Na}_{2} \mathrm{~S}_{2} \mathrm{O}_{3}$ solution in the course of synthesis, the major plasmon bands of prepared AuNPrs located at $808 \mathrm{~nm}$, which were highly suitable for PTT in vivo and in vitro.

As shown in Fig. 1(a), the prepared AuNPrs had three congruent edge lengths of $110 \mathrm{~nm}$, the highly anisotropic shape of flat triangle AuNPrs exhibited a strong absorption in the NIR. To enhance the AuNPrs' stability for further application, prepared AuNPrs were modified with PEG heterobifunctional (-HS and$\mathrm{COOH}$ groups) polyethyleneglycol chains (HS-PEGCOOH, M.W. $5000 \mathrm{~g} / \mathrm{mol}$ ), and then centrifuged for three times, final products were collected for further usage.

\section{Effects of AuNPrs on CIK cells and MGC803 cells}

Cytotoxicity studies are designed to investigate whether the PEGylated AuNPrs are relatively safe for cells. We measured the cell viability by CCK- 8 assay kit after co-incubating the prepared AuNPrs with CIK cells and MGC803 cells for 24 hrs, respectively. The concentration of AuNPrs varied from $10 \mu \mathrm{g} / \mathrm{ml}$ to $200 \mu \mathrm{g} / \mathrm{ml}$, the viability of control group without any treatment is defined to be $100 \%$. As shown in Fig. 2, the prepared AuNPrs displayed very low cytotoxicity even at the highest concentration $(200 \mu \mathrm{g} / \mathrm{ml})$ with the cell viability is $78 \%$ for CIK cells and $75 \%$ for MGC 803 cells, therefore, we considered that prepared 


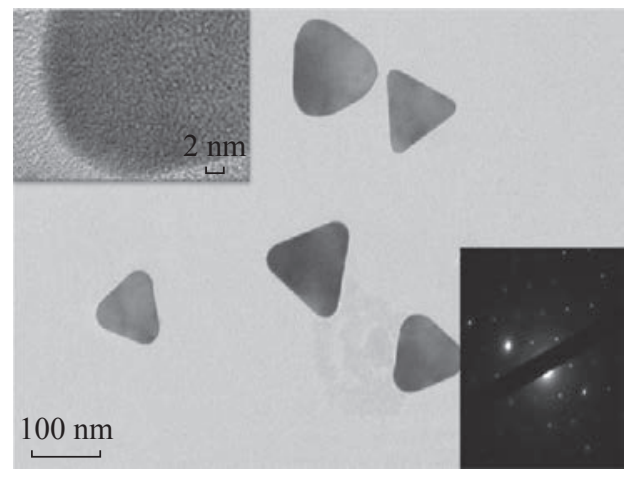

(a)

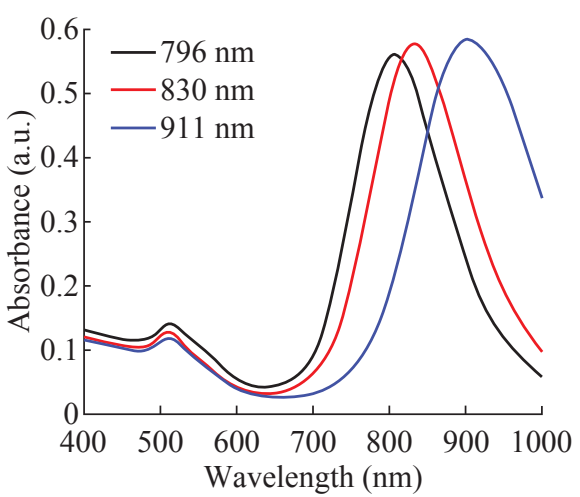

(b)

Fig. 1 Characterization of AuNPrs. (a) TEM images of AuNPrs. (b) UV-Vis-NIR absorbance spectra of AuNPrs, the volume of the second reducing agent is $1.5,2,2.5 \mathrm{~mL}$, respectively.

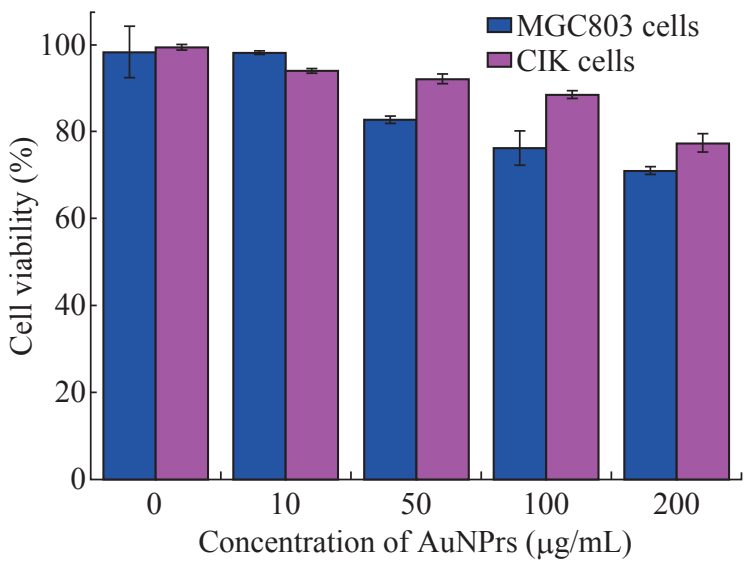

Fig. 2 Viability of MGC803 cells and CIK cells after coincubated with different concentrations of PEGylated AuNPs.

AuNPrs within the dose scope of $200 \mu \mathrm{g} / \mathrm{ml}$ were safe for experiments in cells and animals.

\section{Preparation and characterization of CIK cells labeled with PEGylated Au NPrs}

After PEGylated Au NPrs incubated with human CIK cells for $24 \mathrm{hrs}$, we observed that Au NPrs could enter and locate inside human CIK cells. As shown in Fig. 3(a), plasmon-enhanced two-photon luminescence (TPL) experiment confirmed that PEGylated AuNPrs located inside CIK cells, and that bright golden fluorescence color were distributed inside the cytoplasm. The cellular uptake amount of PEGylated AuNPrs was measured by ICP-MS. As shown in Fig. 3(b), the amount of PEGylated AuNPrs uptaken by CIK cells increased proportionally as the co-incubation time increased. After incubation for $24 \mathrm{hrs}$, the amount of PEGylated AuNPrs uptaken by CIK cells reached 3000 $\mathrm{ng} / 10^{6}$ cells, our results fully demonstrated that human CIK cells could be labeled with PEGylated Au NPrs.

\section{Effects of CIK cells labeled with PEGylated AuNPrs on MGC803 cells}

It is well known that CIK cells are a heterogeneous subset of ex-vivo expanded T lymphocytes, which present a mixed T-NK phenotype and are endowed with a MHC-unrestricted antitumor activity [6][12]. The apoptosis and cell cycle of MGC803 cells were detected by flow cytometry after MGC803 cells co-incubated with CIK cells. As shown in Fig. 4, the MGC803 cells' apoptosis rate displayed the characteristic of time dependence and concentration dependence. After co-incubation of the CIK cells with MGC803 cells by ratio of 1:20 and 1:40 for $12 \mathrm{hrs}$ and $24 \mathrm{hrs}$ respectively, the MGC 803 cells displayed a degree of apoptosis up to $25.9 \%$. Comparing with the control group, the MGC803 cells' cycle distribution changed with some regulation after the same treatment. The number of cells in G1 phase increased $19.49 \%$; decreased $7.81 \%$ and $11.68 \%$ in $\mathrm{G}_{2}$ phase and in $\mathrm{S}$ phase respectively, which highly suggests that the proliferation of MGC803 cells was mainly inhibited in $\mathrm{G}_{1}$ phase, which demonstrate that human CIK cells could inhibit the growth of MGC803 cells via induced apoptosis and G1 phase arrest. We also used human CIK cells labeled with PEGylated AuNPrs to incubate with MGC 803 cells for 24 hrs, we also observed the same inhibition phenomena, which showed that PEGylated AuNPrs did not affect the function of labeled CIK cells.

\section{CIK cells labeled with PEGylated AuNPrs for targeted fluorescent imaging of gastric cancer}

Some reports show that human CIK cells can target in vivo tumor tissues [17]-[22]. However, a lot of reports showed that CIK cells kill tumor cells via 


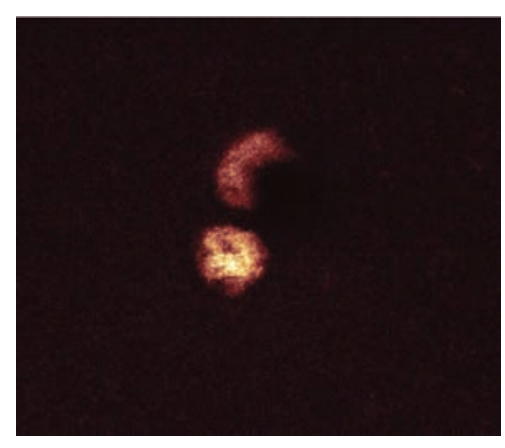

(a)

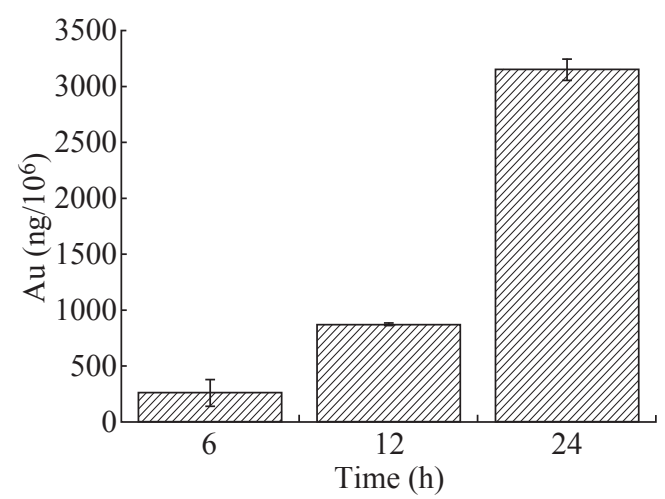

(b)

Fig. 3 Cellular uptake of AuNPrs. (a) Two-photon-laser scanning confocal microscopy image of AuNPrs in CIK cells. (b) Quantification of Au levels by ICP-MS in CIK cells treated with AuNPrs.

(a)

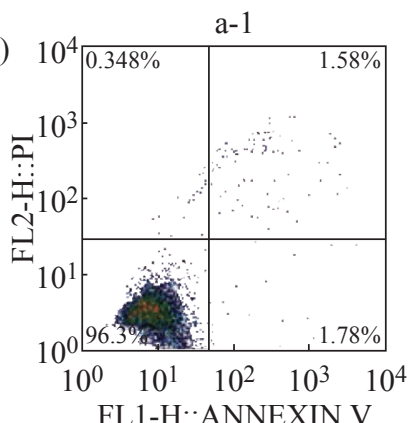

(b)

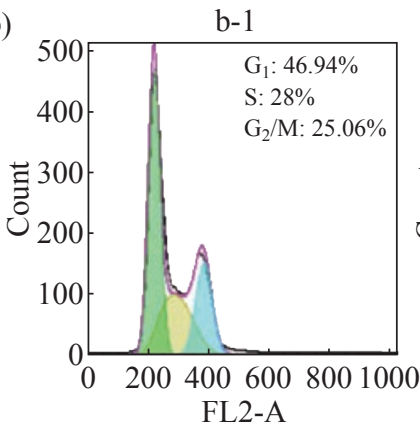

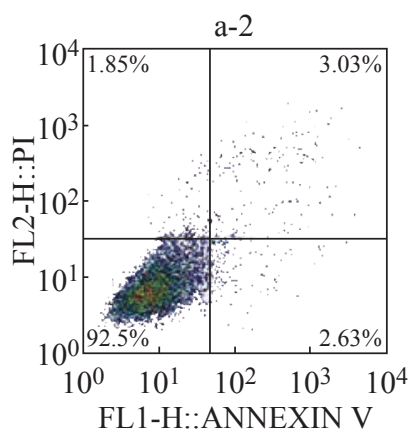

FL1-H::ANNEXIN V

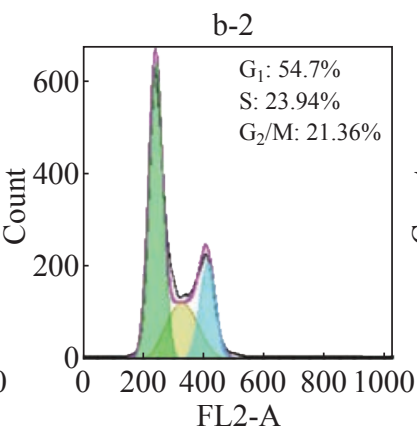

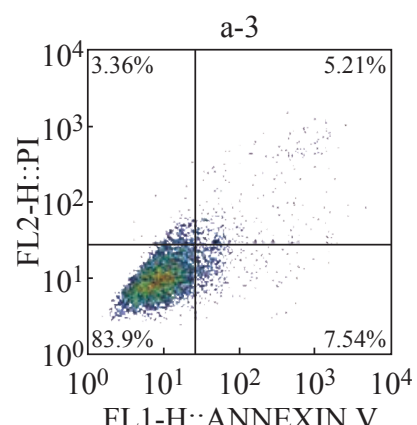

FL1-H::ANNEXIN V

b-3

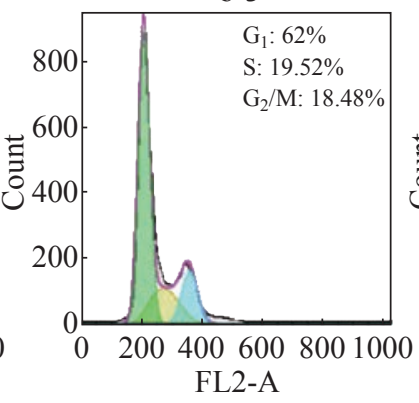

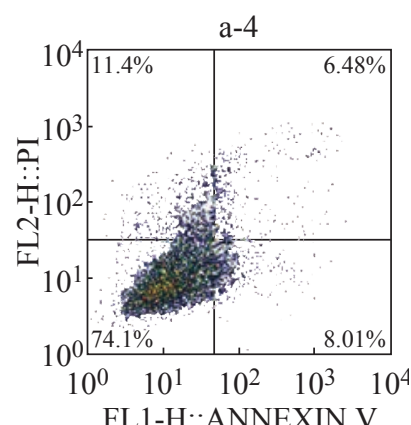

FL1-H::ANNEXIN V

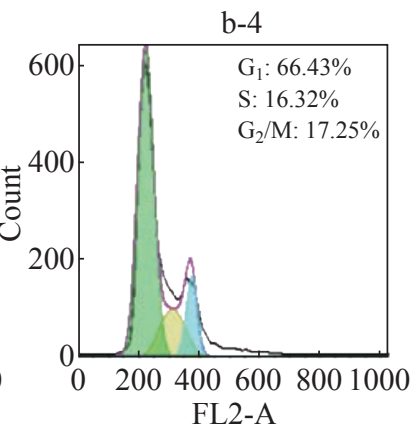

Fig. 4 Flow cytometry analysis of (a) apotosis/necrosis and (b) cell cycle phase distribution in MGC-803 cells after exposure to CIK cells (a-2, b-2) E:T=1:20, 12 hrs; (a-3, b-3) E:T=1:20, 24 hrs; and E:T=1:40, 24 hrs, respectively. (a-1, b-1) cells without any treatment as control.

releasing some special factors $[47,48]$. Up to date, few reports provided clear imaging data that display the course of human CIK cells targeted tumor in vivo. As shown in Fig. 5(a) and 5(b), co-incubated the MGC803 cells with human CIK cells labeled with PEGylated AuNPrs or not for $5 \mathrm{hrs}$; most of human CIK cells have a tendency to attach to MGC803 cells.

In order to clarify whether CIK cells can target in vivo gastric cancer cells, we incubated CIK cells labeled with PEGylated AuNPrs with DiR dye for 24 hrs, and then injected prepared CIK cells into gastric cancer-bearing nude mice model via tail vessel. Then we used animal imaging system to observe the distribution of CIK cells labeled with PEGylated AuNPrs and DiR dye. As shown in Fig. 5(c), one day after injection, CIK cells labeled with PEGylated AuNPrs and DiR dye were mainly distributed in heart, lung, liver and intestinal tract; no CIK cells located inside brain tissues; the tumor site had a weak fluorescent signal. At day 3 post-injection, strong fluorescence signal only located inside tumor site; very less fluorescent signal was observed in other organs, which fully demonstrated that the CIK cells labeled with PEGylated AuNPrs can target in vivo gastric cancer tissues. On day 7 post-injection, the CIK cells labeled with PEGylated AuNPrs still located in the 
(a)

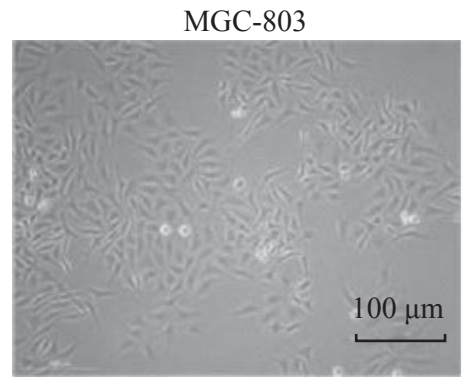

MGC-803/CIK

(b)
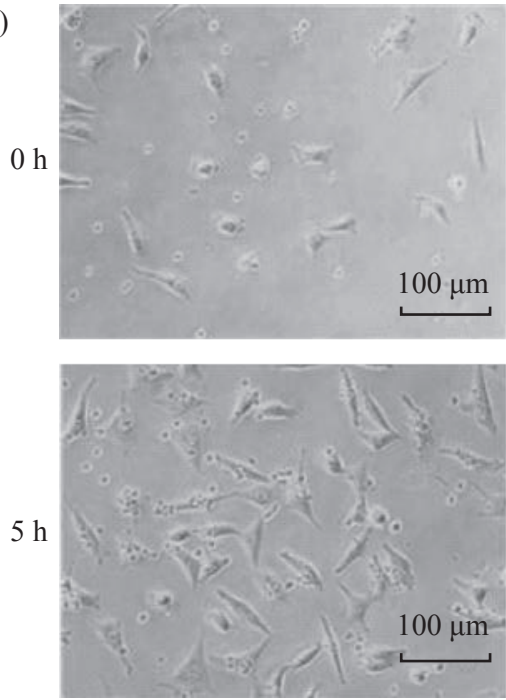

(c)

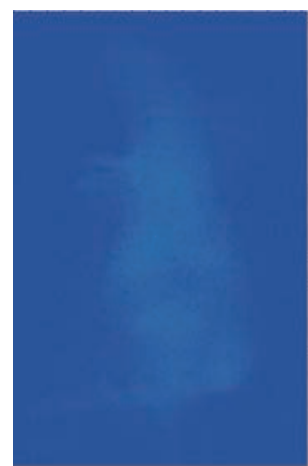

control

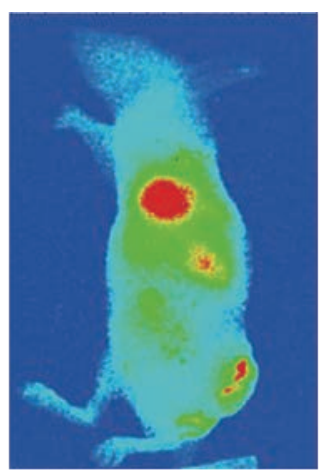

1d

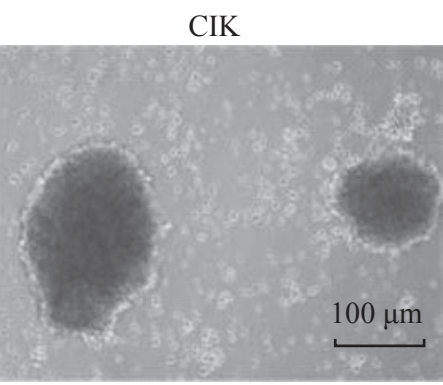

MGC-803/CIK-PEGylated AuNPrs
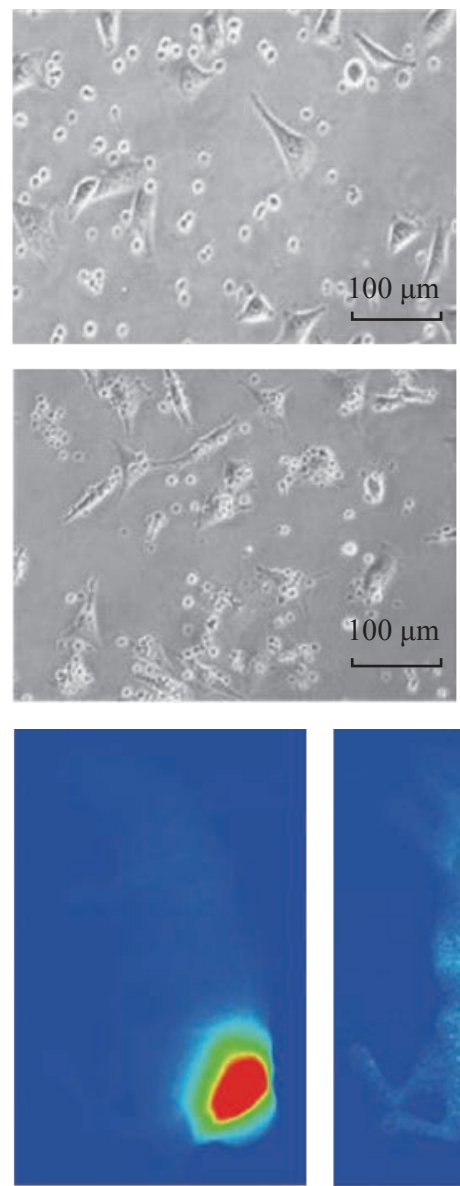

$3 d$

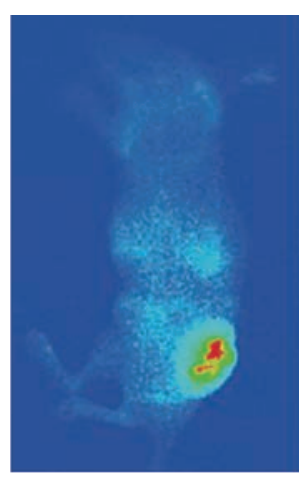

$7 d$

(d)

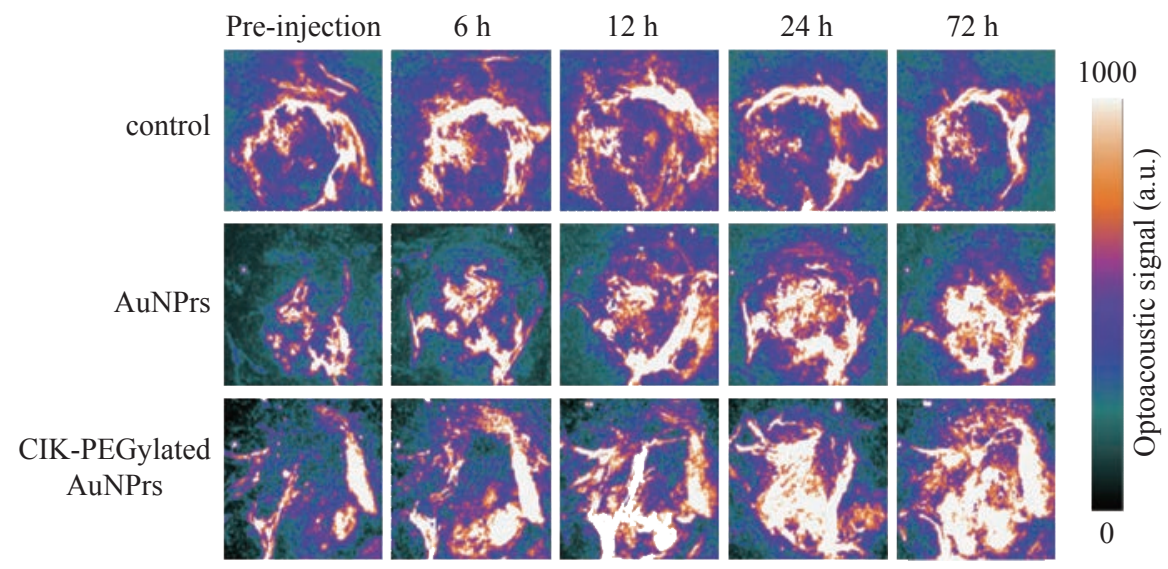

Fig. 5 The cancer-targeted ability of CIK cells (X 50). (a) MGC-803 cells on logarithmic phase and CIK cells cultured for 14 days. (b) Microscope photos of the CIK cells co-incubated with MGC-803 cells at the time point of 0 and 5 hrs. (c) microscope photo after coincubated the CIK-PEGylated AuNPrs cells with MGC-803 cells for 0 and 5 hrs. (d) In vivo fluorescent images of tumor (MGC-803) bearing mouse with intravenous injection of CIK-PEGylated AuNPrs at different time points. 
tumor site with decreased fluorescence signal (Fig. 5(c)). Our results clearly confirm that human CIK cells can target actively in vivo tumor cells.

\section{Human CIK cells labeled with PEGylated AuNPrs for targeted photoacoustic imaging of gastric cancer}

The Nexus 128 is a rapidly emerging, noninvasive, and high-resolution PA imaging system, which can achieve an isotropic and homogeneous spatial resolution of 200 microns. A NIR pulse laser is served as the excitation source, receive PA signals for threedimensional (3d) image reconstruction $[49,50]$. Human CIK cells labeled with PEGylated AuNPrs was first prepared and applied to PA imaging with the aim of attempting to detect subcutaneous gastric cancer tissues in vivo. The human CIK cells and PEGylated AuNPrs injected groups were established as control. When investigating the PA imaging of the tumor site in vivo, laser excitation at a wavelength of $808 \mathrm{~nm}$ with moderate laser energy $1 \mathrm{~W} / \mathrm{cm}^{2}$ (irradiation time: 2 mins) was used for PA probe detection, at which wavelength neovascularization $(\mathrm{CNV})$ could be shown and the probe was at higher PA contrast. A control image has to be acquired before the injection, and then an after-injection image was compared with the control image. Pre-injection scans $(0 \mathrm{~h})$ and postinjection scans were both performed $(6,12,24$ and $72 \mathrm{hrs}$ ). The imaging was displayed with UCLA transverse (axial) as shown in Fig. 5(c). After injection of PEGylated AuNPrs and human CIK cells labeled with PEGylated AuNPrs in the first six hours, there was a gradual signal increase. The blood vessel of the subcutaneous tumors could be clearly shown in the sixth hour. In the 6 to $12 \mathrm{hrs}$ after injection, CIK cells labeled with PEGylated AuNPrs not only made the vessel enhancement, but also began to spread into the surrounding tissue and diffusion into the whole tumor, mainly localizing in the perivascular space, which was similar to the previous report $[51,52]$. At the twentyfourth hour, aggregation signal was stronger than the 12 hour. The similar result could be found after injection of PEGylated AuNPrs, however, agglomeration effect was much weaker than the experimental group. The reason may lie in that PEGylated AuNPrs were widely distributed within the body, whereas CIK cells labeled with PEGylated AuNPrs mainly gathered in tumor sites. For the control group (only CIK cells), there was no distinct accumulation of the PA signal. Only slightly signal enhancement of intravascular could be observed at 6 to $12 \mathrm{hrs}$ after injection, as displayed in Fig. 5(c). Our results fully demonstrate that CIK cells labeled with PEGylated AuNPrs can be used for targeted photoacoustic imaging of gastric cancer in vivo.

\section{CIK cells labeled with PEGylated AuNPrs for photothermal therapy of gastric cancer}

It is reported that AuNPrs could be used as nanoheaters, which transform the NIR photons they absorbed into heat and then kill the cancer cells [53]-[55]. It is very necessary to assess the lightthermal conversion efficiency in colloidal solution and in the cells before experiments in animals. 1 $\mathrm{mL}$ colloidal solution of PEGylated AuNPrs, where its LSPR centered at $808 \mathrm{~nm}$ with concentrations of $0,30,50 \mu \mathrm{g} / \mathrm{mL}$ were prepared. Then we survey the elevation of temperature when illuminate the colloidal in a continuous wave $(\mathrm{CW})$ of $808 \mathrm{~nm}$. As shown in Fig. 6, the temperature rose rapidly in the first $500 \mathrm{sec}$., then became gentle later on. The final $\Delta \mathrm{T}$ can reach up to $30^{\circ} \mathrm{C}$ at the concentration of 50 $\mu \mathrm{g} / \mathrm{mL}$, which demonstrate that prepared PEGylated AuNPrs can transform NIR photons into heat energy rapidly. Compared with previous reports [56][58], prepared PEGylated AuNPrs displayed fast photothermal transformation, we also observed, under laser irradiation, PEGylated AuNPrs always kept the whole integrated, unlike gold nanorods which quickly break into small pieces under laser irradiation. This is an obvious advantage of Au NPrs for photothermal therapy.

In order to investigate the photothermal therapeutic effect of prepared human CIK cells labeled with PEGylated AuNPrs, the viability of GFP-MGC803 cells was measured after co-incubation with CIK cells

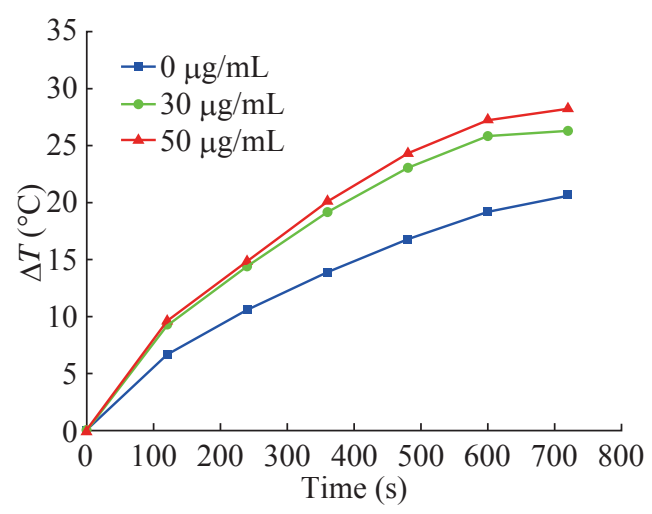

Fig. 6 Temperature traces for colloidal solutions of PEGylated AuNPrs with different concentrations under $808 \mathrm{~nm}$ NIR illumination. 


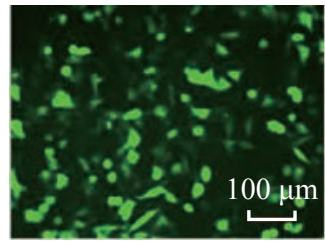

(a)

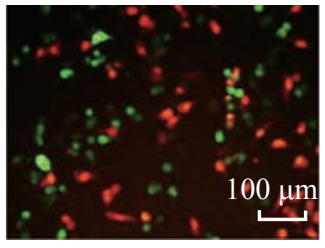

(b)

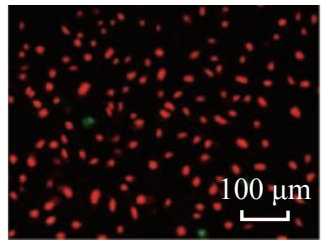

(c)

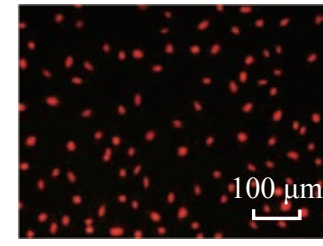

(d)

Fig. 7 Fluorescence images of GFP-MGC803 and PI stained cancer cells. MGC-803 cells were marked by GFP, red stained cells correspond to the impaired cells, whereas green cells are remain viable after irradiation for (a) 0 min, (b) 2 mins, (c) 5 mins, and (d) 10 mins.

labeled with PEGylated AuNPrs $\left(200 \mu \mathrm{g} / 10^{6}\right.$ cells). As shown in Fig. 7(a), the GFP-MGC803 cells coincubated with CIK cells labeled with PEGylated AuNPrs were alive and exhibited green fluorescence signal, after irradiating the cells by using $808 \mathrm{~nm}$ NIR system for different times, the gastric cancer cells showed different degree of impairment, and the dead cells can be stained red with PI, exhibited more and more as the irradiation time increased, as shown in Fig. 7(b) to 7(d), our results fully demonstrate that PEGylated AuNPrs can be used for photothermal therapy of gastric cancer cells in vitro.

In order to observe the photothermal therapeutic effect of human CIK cells labeled with PEGylated AuNPrs in vivo, we prepared gastric cancer-bearing nude mice model, set up control group and test group. For the test group, the mice were injected with human CIK cells labeled with PEGylated AuNPrs via tail vein, and then exposed to $808 \mathrm{~nm}$ laser irradiation at $1 \mathrm{~W} / \mathrm{cm}^{2}$ for $2 \mathrm{mins}$; the control groups included two groups: no.1 control group: injected with PBS only; no. 2 control group: $808 \mathrm{~nm}$ laser irradiation only. All laser irradiation were carried out at 3 day postinjection while the CIK cells with PEGylated AuNPrs accumulated in the tumor site. The temperature variation was monitored by a thermal imager (IR Soft). The results demonstrated that the tumor surface temperature in the test group increased rapidly to $\sim 45$ ${ }^{\circ} \mathrm{C}$ whereas the control group with irradiation only had a slow increase of $\sim 4{ }^{\circ} \mathrm{C}$ (Fig. 8(b)). As shown in Figure $8 \mathrm{c}$, the gastric cancer tissues treated by CIK cells with PEGylated AuNPrs and $808 \mathrm{~nm}$ laser irradiation was remarkably inhibited, a black scar was left at the point of irradiation, and no obvious tumor growth was observed, the control groups had similar tumor growth with a relatively higher speed, as shown in Fig. 8(a), CIK cells could inhibited the growth of gastric cancer in vivo, CIK cells with PEGylated AuNPrs also could inhibit growth of tumor tissues, CIK cells with PEGylated AuNPrs integrated with
$1 \mathrm{~W} / \mathrm{cm}^{2}$ laser irradiation markedly inhibited the growth of gastric cancer in vivo, compared with other groups, there existed statistical difference $(P<0.01)$. Our results show that human CIK cells labeled with PEGylated AuNPrs can target in vivo gastric cancer tissues, combined with laser irradiation, exhibit maximal inhibition therapeutic effect of gastric cancer in vivo.

\section{CIK cells labeled with PEGylated AuNPrs for enhanced immunotherapy}

The serum concentration of cytokine factors were measured in control group, CIK therapy group and photothermal group. As shown in Fig. 9, the levels of cytokine factors such as IFN-g, Il-2, IL-22 and IL-27 in CIK therapy groups rose markedly at day 3 post-injection, as compared with the control group. There existed statistically difference $(P<0.01)$, which highly suggests that the innate immune was enhanced obviously in CIK treatment groups, and that CIK cells can treat tumor via rising the levels of killing tumor cytokine factors such as IFN-g and Il-2.

The serum concentration of cytokine factors were also measured in photothermal group at day 3 postphotothermal therapy. Compared with CIK therapy groups, the concentration of cytokine factors such as IFN-g, Il-2, Il-6, TNF-a, Il-27 rose markedly, compared with CIK therapy group. There existed statistically difference $(P<0.01)$, which highly suggests that Au NPrs-based photothermal therapy can enhance immunotherapy efficacy by raising cytokine factor levels, exhibiting enhanced immuno-photothermal combined therapy.

\section{In vivo distribution and pathological analysis}

We examined biodistribution of CIK cells labeled with PEgylated Au NPrs in gastric cancer-bearing nude mice after intravenous injection. Figure 9 shows 30\% of the injected dose of $\mathrm{Au}$ was found in the blood at 


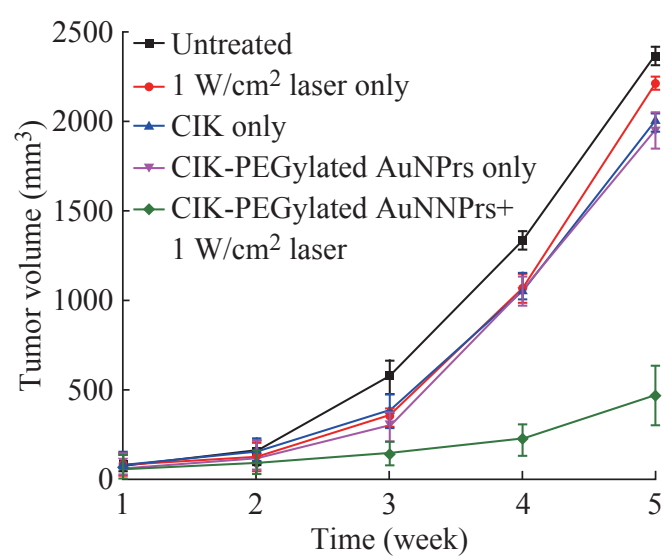

(a)
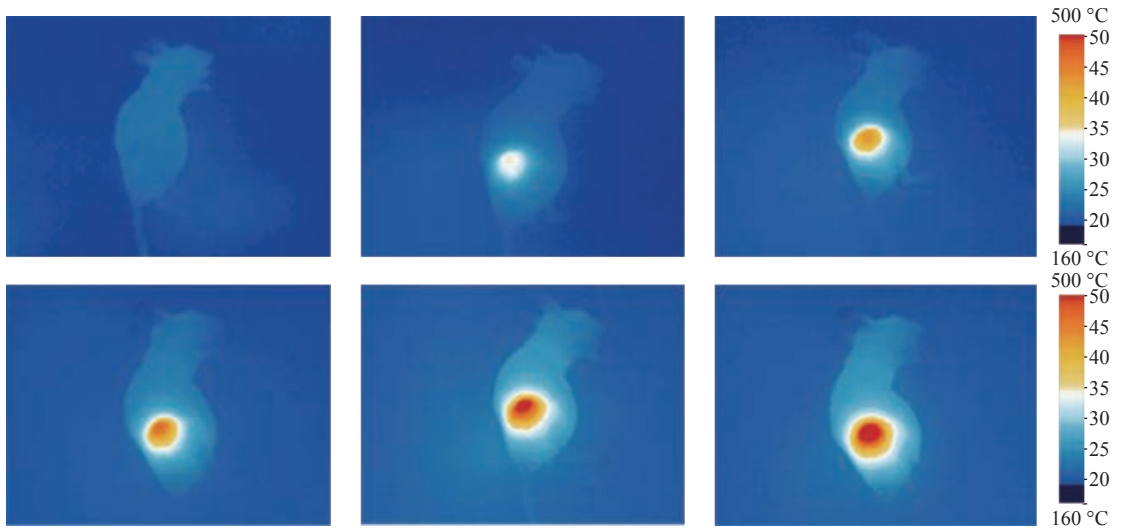

(b)

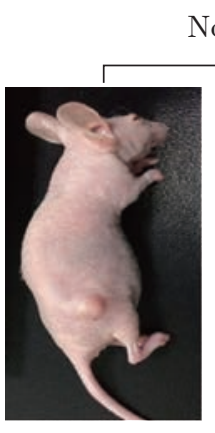

Control
No laser

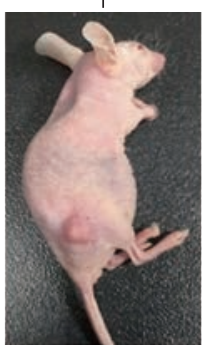

CIK-PEGylated AuNPr

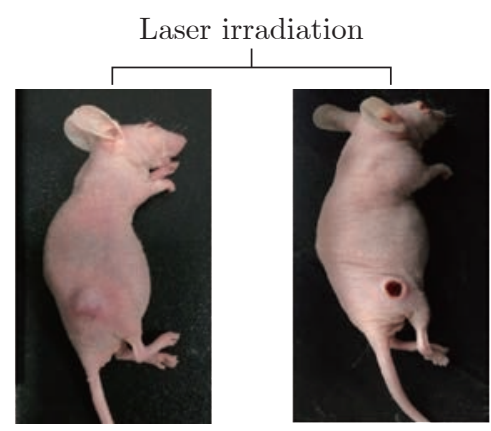

Control
CIK-PEGylated AuNPrs

(c)

Fig. 8 (a) Curve of tumor growth for untreated mice (PBS only), $1 \mathrm{~W} / \mathrm{cm}^{2}$ laser only, CIK-PEGylated AuNPrs only and CIKPEGylated AuNPrs under $1 \mathrm{~W} / \mathrm{cm}^{2}$ laser irradiation. Laser wavelength $=808 \mathrm{~nm}$; irradiation time $=5 \mathrm{mins}\left(1 \mathrm{~W} / \mathrm{cm}^{2}\right)$. (b) thermal images of tumor mice when irradiated with $808 \mathrm{~nm}$ laser. (c) Representative photographs of MGC-803 tumor mice after various treatments.

day 1 after the injection, and no Au was detected in brain tissues, while only $10 \%$ or so of $\mathrm{Au}$ was found in tumor; $20 \%$ or so was found in intestinal tissues; $10 \%$ or so was found in liver and spleen tissues; the amount of $\mathrm{Au}$ in the blood decreased in a time-dependent manner. On day 3, 5 and 7 post-injection, $35 \%$ or so of Au was found in tumor, obviously higher than other tissues, which highly suggests that most CIK cells mainly accumulate on the tumor site; CIK cells should be a safe high-efficient delivery system.
We also used HE staining method to examine pathological changes of important organs such as heart, liver, spleen, lung and kidney, as shown in Fig. 10. No obvious pathological lesions could be observed, which demonstrated that CIK cells labeled with PEgylated Au NPrs do not affect important organs' function.

\section{Possible potential mechanism}

Results mentioned above show that human CIK cells labeled with PEGylated Au NPrs could inhibit growth 


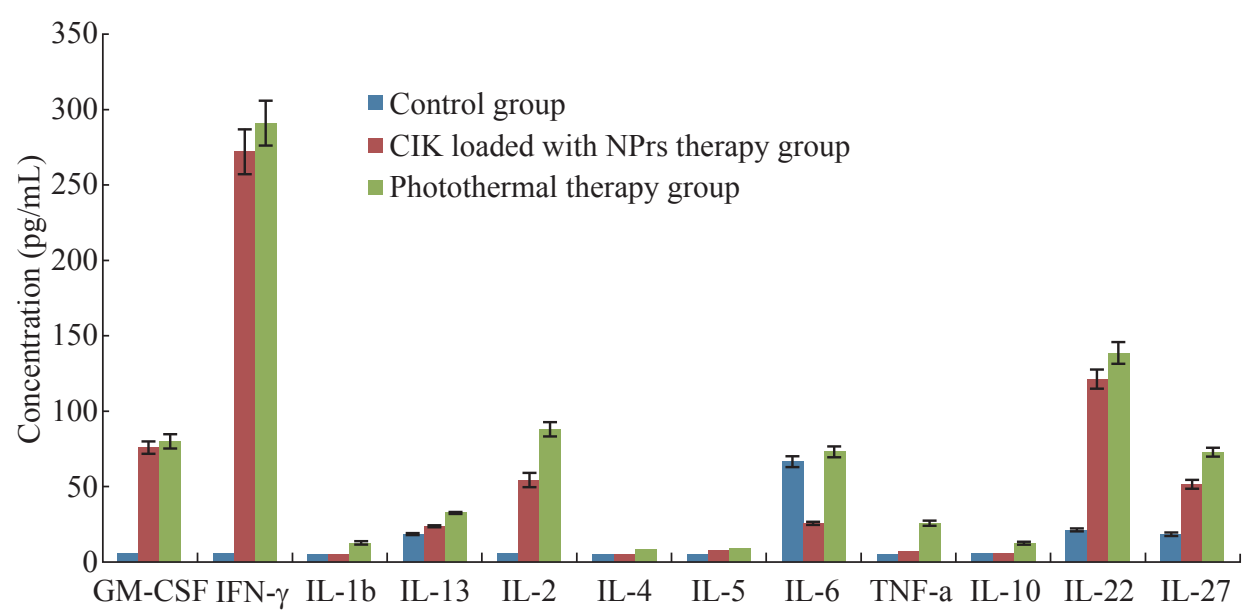

Fig. 9 Serum concentration levels of cytokine factors in control group, CIK loaded with PEGylated Au NPrs and photothermal group.

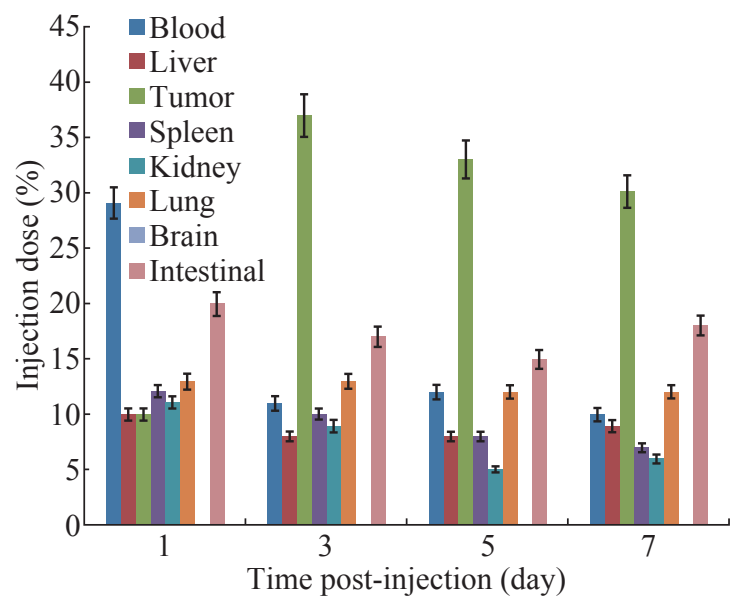

Fig. 10 Distribution of Au NPrs in body at day 1, 3, 5 and 7 day post-injection.

of gastric cancer cells via induced apoptosis and G1 phase arrest; Au NPrs did not affect the CIK cells' therapeutic function. We also observed that gastric cancer cells co-incubated with CIK cells labeled with PEGylated Au NPrs; under $808 \mathrm{~nm}$ laser irradiation for 2 mins, gastric cancer showed death rapidly, presenting strong photothermal therapeutic efficacy. Animal experiments also demonstrated that on day 3 postinjection, most CIK cells labeled with PEGylated Au NPrs gathered in the gastric cancer site; on day 3 to 7 post-injection, most CIK cells still located around the gastric cancer site; less CIK cells were distributed in other organs such as brain, heart, liver, spleen, lung and kidney; therefore, CIK cells labeled with PEGylated $\mathrm{Au}$ NPrs could target in vivo tumor cells actively. Due to that CIK cells are one kind of immunological cells, macrophage cells can not engulf them; therefore, their distribution in liver and spleen organs are very less. Because of the bigger size, CIK cells labeled with
PEGylated Au NPrs can not get through blood-brain barrier; therefore, no CIK cells labeled with PEGylated $\mathrm{Au}$ NPrs located inside brain tissues. Our previous results showed that CXCL12-CXCR4 and CCL19CCR7 loops were involved in the course of MSCs cells targeting in vivo gastric cancer tissues [59]; CIK cells also displayed positive-expression of CXCR4 and CXCR 7; therefore, we predict that CXCL12-CXCR4 and CCL19-CCR7 loops may be involved in the course of CIK cells targeting gastric cancer in vivo. Some chemokine factors may also involve in this course. The levels of cytokine factors such as IFN-g, Il-2, IL22 and IL-27 in CIK therapy groups at day 3 postinjection rose markedly, compared with control group; there existed statistically difference $(P<0.01)$, which highly suggests that the innate immune was enhanced obviously in CIK treatment groups, CIK cells can treat tumor via rising the levels of killing tumor cytokine factors such as IFN-g and Il-2. The serum concentration of cytokine factors also were measured in photothermal group at 3 day post-photothermal therapy. Compared with CIK therapy groups, the concentration of cytokine factors such as IFN-g, Il-2, Il-6, TNF-a, Il-27 rose markedly, compared with CIK therapy group, there existed statistically difference $(P$ $<0.01$ ), which highly suggests that Au NPrs-based photothermal therapy can enhance immunotherapy efficacy by raising cytokine factor levels, exhibiting enhanced immuno-photothermal combined therapy.

Our results also confirm that CIK cells labeled with PEGylated Au NPrs could be used for photoacoustic imaging of in vivo gastric cancer, which clearly display tumor vessels and can therefore be used for diagnosis of early gastric cancer. Our results also show that CIK cells labeled with PEGylated Au NPrs realized 
immunotherapy and photothermal therapy of gastric cancer, exhibiting synergistic therapeutic function and enhancing immunotherapeutic efficacy of CIK cells. The potential mechanism is as follows [60][63]: CIK cells expressed LFA-1 (lymphocyte function associated antigen1), gastric cancer cells expressed ICAM-1(intercellular surface adhesion molecule-1), after LFA-1 binding with ICAM-1, CIK cells released toxic particles: $\mathrm{n}$ - methyl carbonyl sulfur - left-handed - lysine methyl ester (BLT), killing tumor cells; CIK cells expressed CD3 and CD 56, CD3 combined with T cell receptor, activated T cells, inducing TCR-mediated specific cytotoxicity to gastric cancer cells, finally killing tumor cells in vivo. Au NPrs inside CIK cells produced heat energy under $808 \mathrm{~nm}$ laser irradiation for 2 mins, and denatured gastric cancer cells and induced necrosis of gastric cancer cells, forming small scar fiber tissues. In the course of photothermal therapy of Au NPrs, CIK cells were stimulated by heart energy, secreted a lot of chemokine factors, and enhanced therapeutic efficacy of gastric cancer. The schematic of possible mechanism is as shown in Fig. 12.
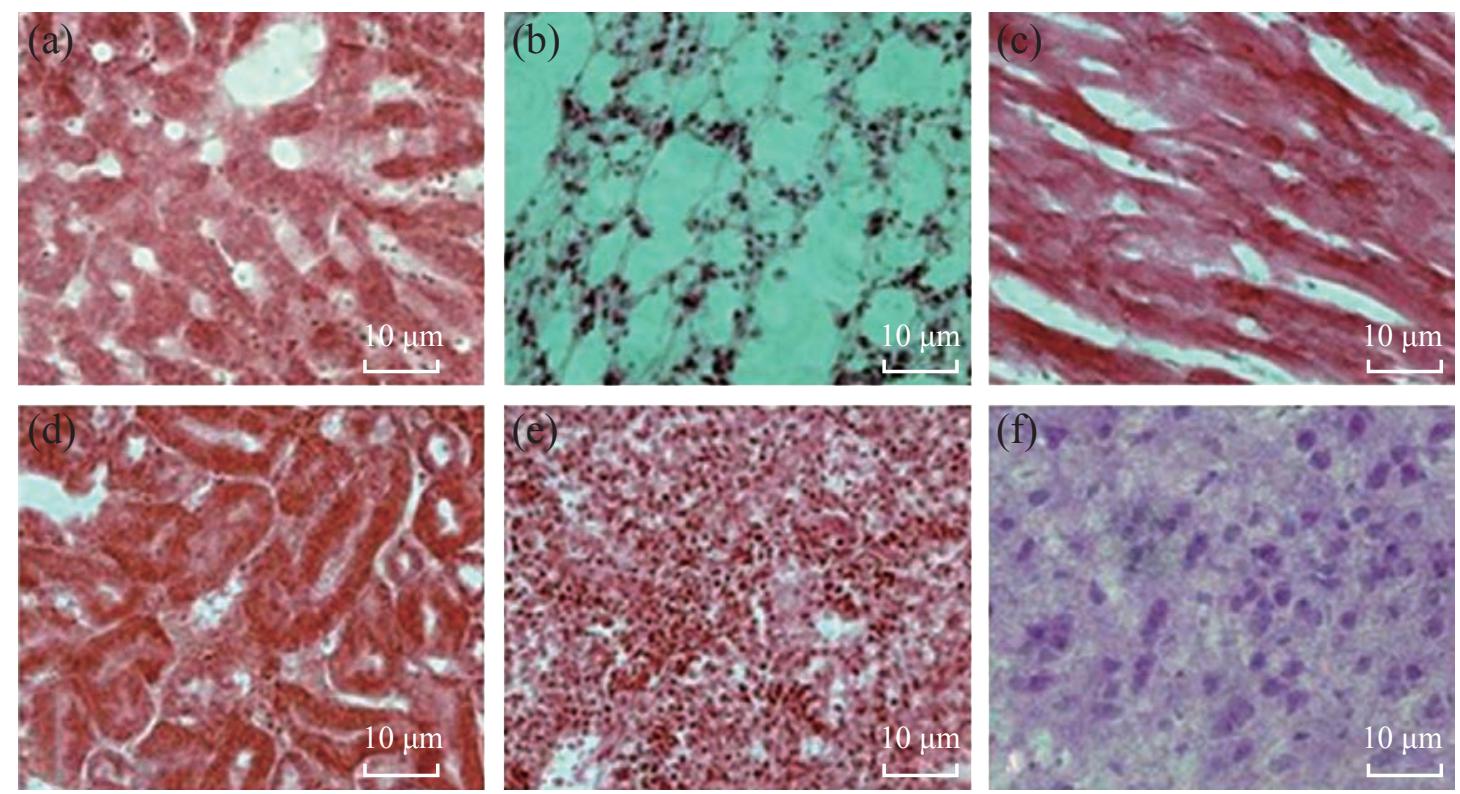

Fig. 11 Histological imagines $\left(20^{\prime}\right)$ of different tissues using hematoxylin and eosin staining at 7 day post-injection: (a) Liver; (b) Lung; (c) Heart; (d) Kidney; (e) Spleen; and (f) Brain.
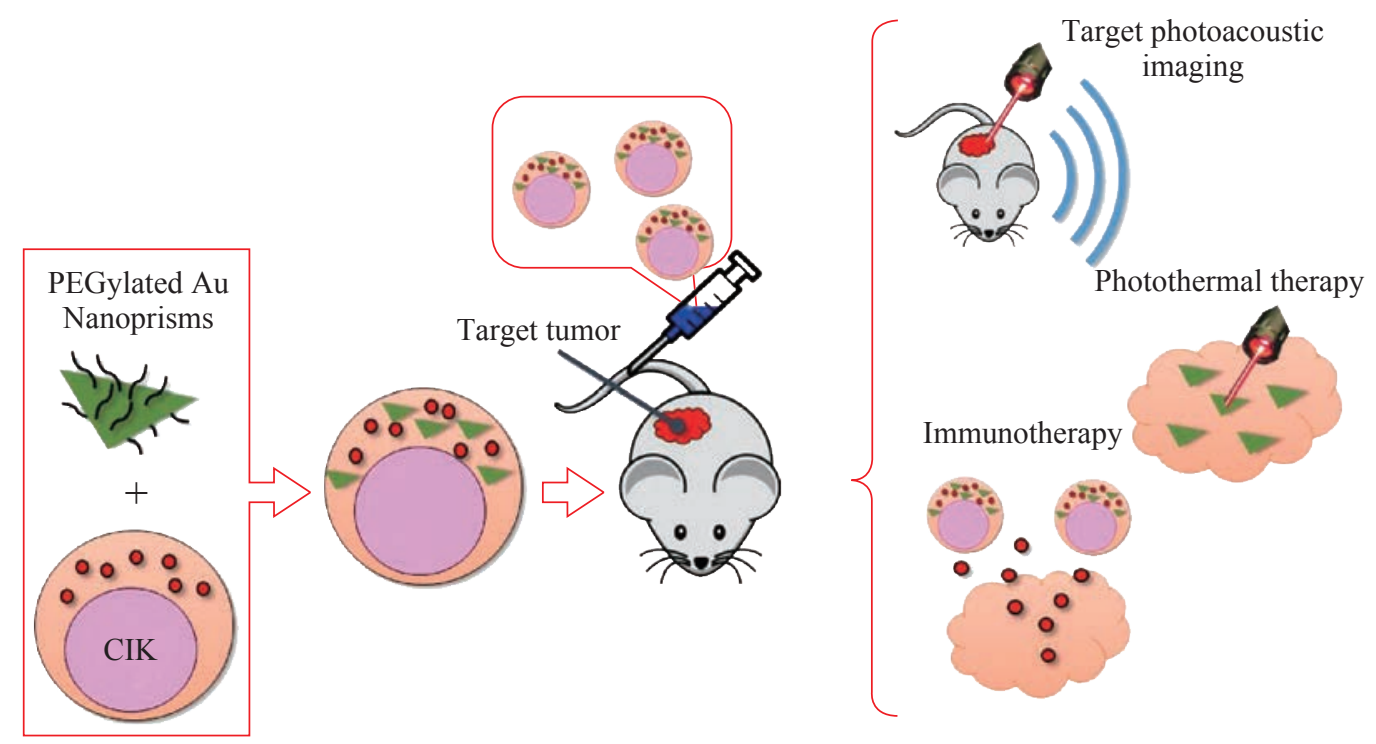

Fig. 12 Schematic of the proposed mechanism of CIK labeled with PEGylated gold nanoprisms for targeted photoacoustic imaging, immunotherapy and photothermal therapy. 


\section{Conclusions}

In summary, we successfully prepared for the first time CIK cells with PEGylated AuNPrs and realized gastric cancer targeted photoacoustic imaging, enhanced immunotherapy and photothermal therapy. Human CIK cells labeled with PEGylated AuNPrs could target actively and gather in vivo gastric cancer tissues at day 3 post-injection, inhibit the growth of tumor cells in vivo, be combined with photothermal therapy of PEGylated AuNPrs, and exhibit enhanced inhibition of growth of tumor. Especially, PEGylated $\mathrm{Au}$ NPrs did not break into pieces under laser irradiation, and produced long-time heat energy, unlike gold nanords which break into pieces under laser irradiation. Due to that PEGylated AuNPrs own good biocompatibility, Human CIK cells labeled with PEGylated AuNPrs have a clinical translation prospect. Furthermore, human CIK cells with PEGylated AuNPrs designed here is a universal multifunctional theranostic platform. The AuNPrs can be replaced by other nanoparticles with imaging or cancerkilling ability; the human CIK cells in the "CIKNPs" mode act as a "vehicle", carrying functional nanoparticles or drugs accurately to the tumor site. Development of human immunocells-based delivery sytem should be a good direction to solve current drug delivery system' synthesis and toxicity. Human CIK cells with PEGylated AuNPrs have great potential in applications such as targeted photoacoustic imaging, immunotherapy and photothermal therapy of gastric cancer and other tumors, as well as high efficient delivery platform for gene or drugs in near future.

\section{Authors claim that they have no competing interests.}

\section{Acknowledgements}

This work was supported by Chinese Key Basic Research Program (973 Project) (No. 2010CB933901 and 2015CB931802), the National Natural Scientific Foundation of China (Grant No. 81225010, 81327002, and 31170961), 863 project of China (no.2012AA022703 and 2014AA020700), and Shanghai Science and Technology Fund (No. 13NM1401500). We would like to thank the Instrumental Analysis Center of SJTU for the TEM analysis, dark-field imaging and ICP measurements.

\section{References}

[1] J. Ferlay, H.R. Shin, F. Bray, et al., Estimates of worldwide burden of cancer in 2008: GLOBOCAN 2008. Int. J. Cancer, 2010, 127: 2893-2917.

[2] R. Siegel, D. Naishadham and A. Jemal, Cancer statistics, 2013. CA Cancer J Clin, 2013, 63: 11-30.

[3] T. Takahashi, Y. Saikawa and Y. Kitagawa, Gastric cancer: current status of diagnosis and treatment. Cancers (Basel), 2013, 5: 48-63.

[4] I. Proserpio, S. Rausei, S. Barzaghi, et al., Multimodal treatment of gastric cancer. World journal of gastrointestinal surgery, 2014, 6: 55-58.

[5] D. Zhang, D. Fan, New insights into the mechanisms of gastric cancer multidrug resistance and future perspectives. Future Oncol, 2010, 6: 527-537.

[6] D. Cui, L. Zhang, X.J. Yan, et al., A microarraybased gastric carcinoma prewarning system. World $J$ Gastroenterol, 2005, 11: 1273-1282.

[7] F.C. Schmeel, L.C. Schmeel, S.M.Gast, et al., Adoptive immunotherapy strategies with cytokine-induced killer (CIK) cells in the treatment of hematological malignancies. International journal of molecular sciences, 2014, 15: 14632-14648.

[8] L. Gammaitoni, L. Giraudo, V. Leuci, et al., Cytokine induced killer cells effectively kill chemo-resistant melanoma cancer stem cells. Journal of translational medicine, 2015, 13(Suppl 1): O1.

[9] E. Rettinger, S. Huenecke, V. Pfirrmann, et al., Repetitive infusions of Cytokine-induced killer (CIK) cells for treatment of impending relapse in high-risk leukemia patients after allogeneic stem cell transplantation. Blood, 2014, 124(21): 2438-2438.

[10] D. Sangiolo, G. Mesiano, L. Gammaitoni, et al., Cytokine-induced killer cells eradicate bone and softtissue sarcomas. Cancer researchI, 2014, 74: 119-129.

[11] C. Li, S. Liang, C. Zhang, et al., Allogenic dendritic cell and tumor cell fused vaccine for targeted imaging and enhanced immunotherapeutic efficacy of gastric cancer. Biomaterials, 2015, 54: 177-187.

[12] J.K. Chan, C.A. Hamilton, M.K. Cheung, et al., Enhanced killing of primary ovarian cancer by retargeting autologous cytokine-induced killer cells with bispecific antibodies: a preclinical study. Clinical Cancer Research, 2006, 12: 1859-1867.

[13] Z. Zhang, L.P. Wang, Z.Z. Luo, et al., Efficacy and safety of cord blood-derived cytokine-induced killer cells in treatment of patients with malignancies. Cytotherapy, 2015, 17: 1130-1138.

[14] X. Zhao, Z. Zhang, H. Li, et al., Cytokine induced killer cell-based immunotherapies in patients with different stages of renal cell carcinoma. Cancer Letter, 2015, 362: 192-198.

[15] C.E. Jaekel, I.H. Schmidt-Wolf, An update on new adoptive immunotherapy strategies for solid tumors with cytokine-induced killer cells. Expert Opinion on Biological Therapy, 2014, 14: 905-916.

[16] G. Mesiano, M. Todorovic, L. Gammaitoni, et al., Cytokine-induced killer (CIK) cells as feasible and effective adoptive immunotherapy for the treatment of solid tumors. Expert Opinion on Biological Therapy, 2012, 12: 673-684.

[17] J. Zhang, Y. Yang, H. Fu, et al., CIK cell therapy for solid tumor. Nano Biomed. Eng., 2014, 6(2): 60-66.

[18] I. Venugopal, S. Pernal, T. Fusinatto, et al., Quantum dot conjugated magnetic nanoparticles for targeted drug delivery and imaging. Nano Biomed. Eng., 2016, 8(1): 2438.

[19] J.S. Xu, Y. Cao, C.Y. Xu, et al., Phase-transition perfluorocarbon nanoparticles for ultrasound molecular imaging and therapy. Nano Biomed. Eng., 2015, 7(1): 8-19.

[20] B. Shen, Systems molecular imaging: right around the 
corner. Nano Biomed. Eng., 2014, 5(1): 1-5.

[21] Y.S. Chen, D.X. Cui, Non-spherical gold nanoparticles: tumor imaging and therapy. Nano Biomed. Eng., 2013, 5(4): 153-160.

[22] Y. Yang, J. Zhang, F. Xia, et al., Human CIK cells loaded with $\mathrm{Au}$ nanorods as a theranostic platform for targeted photoacoustic imaging and enhanced immunotherapy and photothermal therapy. Nanoscale Res. Lett., 2016, 11: 285

[23] D. Cui, Y. Yang, J. Zhang, et al., Gold nanoprims as theranostic platform for enhanced drug delivery, targeted imaging, and photothermal therapy. Nanomedicine, 2016, 12: 456 .

[24] Y.L. Liu, M. Yang, J.P. Zhang, et al., Human induced pluripotent stem cells for tumor targeted delivery of gold nanorods and enhanced photothermal therapy. ACs Nano, 2016, 10: 2375-2385.

[25] C.C. Bao, J. Conde, J. Curtin, et al., Bioresponsive antisense DNA gold nanobeacons as a hybrid in vivo theranostics platform for the inhibition of cancer cells and metastasis. Sci. Rep., 2015, 5: 12197.

[26] D.X. Cui, C.L. Zhang, B. Liu, et al., Regression of gastric cancer by systemic injection of RNA nanoparticles carrying both ligand and siRNA. Sci. Rep., 2015, 5: 10726

[27] J. Conde, C.C. Bao, Y.Q. Tan, et al., Dual targeted immunotherapy via in vivo delivery of biohybrid RNAiPeptide nanoparticles to tumor-associated macrophages and cancer cells. Adv. Fun. Mater., 2015, 25: 4183-4194.

[28] J. Lin, S.J. Wang, P. Huang, et al., Photosensitizer-loaded gold vesicles with strong plasmonic coupling effect for imgaing-guided photothermal/ photodynamic therapy. ACs Nano, 2013, 7: 5320-5329.

[29] I.R. Corbin, J. Chen, W. Cao, et al., Enhanced cancertargeted delivery using engineered high-density lipoprotein-based nanocarriers. Journal of Biomedical Nanotechnology, 2007, 3: 367-376.

[30] P.Huang, L.Bao, C.Zhang, et al. Folic acid-conjugated silica-modified gold nanorods for X-ray/CT imagingguided dual-mode radiation and photo-thermal therapy. Biomaterials, 2011, 32: 9796-9809.

[31] J.H. Park, G. von Maltzahn, M.J. Xu, et al., Cooperative nanomaterial system to sensitize, target, and treat tumors. Proceedings of the National Academy of Sciences, 2010, 107: 981-986.

[32] W. Lu, Q. Huang, G. Ku, et al., Photoacoustic imaging of living mouse brain vasculature using hollow gold nanospheres. Biomaterials, 2010, 31: 2617-2626.

[33] S.J. Liang, C. Li, C.L. Zhang, et al., CD44 monoclonal antibody-conjugated gold nanostars for targeted photoacoustic imaging and plasmonic photothermal therapy of gastric cancer stem-like cells. Theranostics, 2015, 5: 970-984.

[34] C.J. Orendorff, T.K. Sau and C.J. Murphy, Shapedependent plasmon-resonant gold nanoparticles. Small, 2006, 2: 636-639.

[35] C. Bao, J. Conde, E. Polo, et al., A promising road with challenges: where gold nanoparticles are in translationalresearch? Nanomedicine, 2014, 9: 2353-2370.

[36] A. Ambrosone, P. del Pino, V. Marchesano, et al., Gold nanoprisms for photothermal cell ablation in vivo. Nanomedicine (Future Medicine), 2014, 9(13): 19131922.

[37] C.C. Bao, N. Beziere, P. del Pino, et al., Gold nanoprisms as optoacoustic signal nanoamplifiers for in vivo bioimaging of gastrointestinal cancers. Small, 2013, 9: 68-74.

[38] D.P. Yang, D.X. Cui, Advances and prospects of gold nanorods. Chemistry- An Asian Journal, 2008, 3: 2010 2022.

[39] B. Pelaz, P. del Pino, Synthesis and applications of gold nanoparticles. Frontiers of Nanoscience, 2012, 4: 3-33.
[40] B. Pelaz, V. Grazu, A. Ibarra, et al., Tailoring the synthesis and heating ability of gold nanoprisms for bioapplications. Langmuir, 2012, 28: 8965-8970.

[41] B.A. Mehta, S. Wolf, Two pathways of exocytosis of cytoplasmic granule contents and target cell killing by cytokine-induced CD3CD56 killer cells. Blood, 1995, 86: 3493-3499.

[42] G.V. Hartland, G. Schatz, Virtual issue,plasmon resonances-a physical chemistry perspective. The Journal of Physical Chemistry C, 2011, 115: 15121-15123.

[43] X. Huang, I.H. El-Sayed, W. Qian, et al., Cancer cell imaging and photothermal therapy in the near-infrared region by using gold nanorods. Journal of the American Chemical Society, 2006, 128: 2115-2120.

[44] S. Link, M.A. El-Sayed, Shape and size dependence of radiative, non-radiative and photothermal properties of gold nanocrystals. International Reviews in Physical Chemistry, 2000, 19: 409-453.

[45] M.A. Mackey, M.R. Ali, L.A. Austin, et al., The most effective gold nanorod size for plasmonic photothermal therapy: theory and in vitro experiments. The Journal of Physical Chemistry B, 2014, 118: 1319-1326.

[46] S. Ye, G. Marston, J.R. McLaughlan, et al., Engineering gold nanotubes with controlled length and near-infrared absorption for theranostic applications. Advanced Functional Materials, 2015, 25: 2117-2127.

[47] J.V. Jokerst, A.J. Cole, S.E. Bohndiek, et al., Gold nanorods combine photoacoustic and Raman imaging for detection and treatment of ovarian cancer. Proc. SPIE 8943, Photons Plus Ultrasound: Imaging and Sensing 2014. Mar. 3, 2014: 894366.

[48] Y. Li, T. Wen, R. Zhao, et al., Localized electric field of plasmonic nanoplatform enhanced photodynamic tumor therapy. ACS nano, 2014, 8(11): 11529-11542.

[49] N.R. Jana, L. Gearheart and C.J. Murphy, Seed-mediated growth approach for shape-controlled synthesis of spheroidal and rod-like gold nanoparticles using a surfactant template. Advanced Materials, 2001, 13: 1389.

[50] B. Nikoobakht, M.A. El-Sayed, Preparation and growth mechanism of gold nanorods (NRs) using seed-mediated growth method. Chemistry of Materials, 2003, 15: 19571962.

[51] A.M. Alkilany, L.B. Thompson, S.P. Boulos, et al., Gold nanorods: their potential for photothermal therapeutics and drug delivery, tempered by the complexity of their biological interactions. Advanced drug delivery reviews, 2012, 64: 190-199.

[52] J. Park, A. Estrada, K. Sharp, et al., Two-photon-induced photoluminescence imaging of tumors using near-infrared excited gold nanoshells. Optics Express, 2008, 16: 15901599.

[53] N.J. Durr, T. Larson, D.K. Smith, et al., Two-photon luminescence imaging of cancer cells using molecularly targeted gold nanorods. Nano letters, 2007, 7: 941-945.

[54] H. Wang, T.B. Huff, D.A. Zweifel, et al., In vitro and in vivo two-photon luminescence imaging of single gold nanorods. Proceedings of the National Academy of Sciences of the United States of America, 2005, 102: 15752-15756.

[55] C.E. Jäkel, I.G. Schmidt-Wolf, An update on new adoptive immunotherapy strategies for solid tumors with cytokineinduced killer cells. Expert opinion on biological therapy, 2014, 14: 905-916.

[56] R. Nishimura, J. Baker, A. Beilhack, et al., In vivo trafficking and survival of cytokine-induced killer cells resulting in minimal GVHD with retention of antitumor activity. Blood, 2008, 112: 2563-2574.

[57] C.L. Zhang, C. Li, Y.L. Liu, et al., Gold nanoclustersbased nanoprobes for simultaneous fluorescence imaging and targeted photodynamic therapy with superior 
penetration and retention behavior in tumors. Adv. Fun. Mater, 2015, 25: 1314-1325.

[58] Z.M. Li, P. Huang, X.J. Zhang, et al., RGD-conjugated dendrimer-modified gold nanorods for in vivo tumor targeting and photothermal therapy. Molcular Pharmaceutics, 2009, 7: 94-104.

[59] J. Ruan, J.J. Ji, H. Song, et al., Fluorescent magnetic nanoparticles-labeled mesenchymal stem cells for targeted imaging and hyperthermia therapy of in vivo gastric cancer. Nanoscale Research Letters, 2012, 7: 309.

[60] M.R. Verneris, M. Kurnacker, V. Mailander, et al., Resistence of ex vivo expanded CD3+CD56+ T cells to Fas-mediated apoptosis. CancerImmunol Immunolther, 2000, 49: 335-345.

[61] M.R.Alderson, T.W. Tough, et.al., Fas ligand mediates activation-induced cell death in human T lymphocytes. Exp Med, 2012, 181: 71
[62] G. Dranoff, Cytokines in cancer pathogenesis and cancer therapy. Nature Reviews Cancer, 2011, 4: 11-22

[63] S. Thanendrarajan, M. Nowak, H. Abeken, et al., Combining cytokine induced killer cells with vaccination in cancer immunotherapy: more than one plus one? Leuk Res., 2011, 35: 1136-1142.

Copyright@ 2016 Jingjing Zhang, Fangfang Xia, Yao Yang, Caixia Yue, Chunlei Zhang, Yuming Yang, Lijun Ma, Gabriel Alfranca, Yanlei Liu, Yafei Hou, Weilin Jin, Jian Ni, Jesus Martinez de la Fuente and Daxiang Cui. This is an openaccess article distributed under the terms of the Creative Commons Attribution License, which permits unrestricted use, distribution, and reproduction in any medium, provided the original author and source are credited. 